\title{
Avaliação do Programa de Educação pelo Trabalho para Saúde - PET-Saúde/Nigilância em Saúde pelos seus atores
}

\author{
Liziane Maahs Flores(a) \\ Ana Luiza Trindade ${ }^{(b)}$ \\ Daiane Rodrigues de Loreto(c) \\ Beatriz Unfer ${ }^{(d)}$ \\ Marinel Mór Dall'Agnol(e)
}

\section{Introdução}

O Programa de Educação pelo Trabalho para a Saúde/Vigilância em Saúde (PET-Saúde/VS) é promovido pelos Ministérios da Saúde e da Educação, no Brasil, e desenvolvido por universidades em parceria com as Secretarias Estaduais e/ou Municipais de Saúde 1 . Este programa foi implementado na Universidade Federal de Santa Maria (UFSM) desde maio de 2013, disponibilizando bolsas para tutores (docentes), preceptores (profissionais dos serviços) e estudantes de graduação da área da saúde ${ }^{2}$, e oportunizando a realização de estudos em vigilância em saúde, ampliando a integração entre serviços de saúde e instituições de Ensino Superior.

A Vigilância em Saúde (VS) constitui um processo contínuo e sistemático de coleta, consolidação, análise e disseminação de dados sobre eventos relacionados à saúde, visando o planejamento e a implementação de medidas de saúde pública para a proteção da saúde da população, a prevenção e o controle de riscos, agravos e doenças, bem como para a promoção da saúde ${ }^{3}$. As ações de VS abrangem toda a população brasileira e envolvem práticas e processos de trabalho voltados, dentre outras questões, para a detecção oportuna e a adoção de medidas adequadas para a resposta às emergências de saúde pública ${ }^{4}$.

Diante do desastre ocorrido em boate de Santa Maria, em 27 de janeiro de 2013, a equipe do PET-Saúde/VS da UFSM direcionou suas atividades ao monitoramento desta situação de emergência. Os serviços de saúde municipais e da $4^{\mathrm{a}}$ Coordenadoria Regional de Saúde do Estado do Rio Grande do Sul (4a CRS) apresentaram suas necessidades por meio de seus preceptores vinculados ao PET e seus gestores. A universidade aceitou os desafios propostos pelos serviços de saúde e auxiliou na formulação e execução de projetos por meio de seus docentes (tutores) e estudantes de cursos de graduação da área da saúde. Após um ano desta integração ensino-serviço, em março de 2014, estudantes, tutores e preceptores decidiram realizar uma avaliação do programa.

Entendendo que a avaliação de programas deve estar presente no planejamento, na implementação e no funcionamento dos serviços e instituições ${ }^{5}$, elaborou-se uma ficha para avaliar o PET-Saúde/VS da UFSM, e aplicou-se em toda a equipe. "Avaliar" significa o ato de determinar a qualidade,

${ }^{(a, e)}$ Departamento de Saúde da Comunidade, Centro de Ciências da Saúde (CCS), Universidade Federal de Santa Maria (UFSM). Av. Roraima, 1000 Prédio 26 (CCS), sala 1244, Camobi. Santa Maria, RS, Brasil. 97105-900. lizianemf@gmail.com marinelmd@terra.com.br

(b) Acadêmica, curso de Medicina, CCS, UFSM. Santa Maria, RS, Brasil. atrindade93@gmail.com

(c) Acadêmica, curso de Farmácia CCS, UFSM. Santa Maria, RS, Brasil. daiadeloreto@hotmail.com

(d) Departamento de Estomatologia, CCS, UFSM. Santa Maria, RS, Brasil. unfer@terra.com.br 
o valor e o mérito de algo, resultando, por consequência, em qualificação do conhecimento científico ou prático a respeito do tema estudado ${ }^{5}$. Segundo o $\mathrm{DeCS}^{6}$, "avaliação em saúde" é um processo crítico-reflexivo, contínuo e sistemático sobre práticas e processos desenvolvidos no âmbito da saúde, sintetizados por indicadores de natureza quantitativa e/ou qualitativa, a fim de proporcionar informações para auxiliar processos de tomada de decisão.

Neste sentido, a avaliação do PET-Saúde/VS da UFSM objetivou conhecer as principais dificuldades e facilidades vivenciadas, e as contribuições do programa para o ensino e/ou para o serviço. Este artigo pretende descrever e compartilhar esta experiência e discutir os resultados obtidos nesta avaliação, por meio de análise qualitativa, a fim de subsidiar o planejamento e a programação da etapa final do PETSaúde/VS da UFSM.

\section{Referencial teórico}

O Município de Santa Maria, localizado no centro do Estado do Rio Grande do Sul, a 292 Km de Porto Alegre, configura-se o principal polo assistencial da região. Pelos dados do último censo7, o município tem 261.031 habitantes (95\% urbano). No dia 27 de janeiro de 2013, esta população sofreu o impacto de um evento extremo, o incêndio ocorrido em uma boate, com repercussão e comoção nacional e internacional. Este desastre envolveu, sobretudo, jovens na faixa etária de 16 a trinta anos, e caracterizou-se como o segundo maior desastre em número de óbitos em incêndio ocorrido no país 8 . Por se tratar de uma situação de alta complexidade, com necessidade de intervenção imediata, de médio e longo prazo, este momento requereu uma rigorosa articulação estratégica de políticas públicas, de diversas instituições e segmentos envolvidos, responsáveis pela definição, encaminhamento e implementação de medidas preventivas e de intervenções.

O PET-Saúde/VS da UFSM foi estruturado a fim de realizar a análise situacional da saúde no município de Santa Maria. Diante do desastre ocorrido na boate, incluiu, em seus objetivos, realizar ações de vigilância emergenciais às vítimas diretas e indiretas. Nesse sentido, o PET-Saúde/VS se destina: a viabilizar o aperfeiçoamento e a especialização em serviço, bem como a iniciação ao trabalho, os estágios, as pesquisas e vivências, por meio da integração ensino-serviço, considerando as necessidades do SUS na área de vigilância em saúde 1 .

A integração ensino-serviço é o trabalho coletivo, pactuado e integrado de estudantes e professores dos cursos de formação na área da saúde com profissionais que compõem as equipes dos serviços de saúde, incluindo-se os gestores, visando à qualidade de atenção à saúde individual e coletiva, à qualidade da formação profissional e à satisfação dos profissionais dos serviços ${ }^{9}$. A integração ensinoserviço surge, então, como mediadora de diálogos, reflexões e aprendizados múltiplos, em que a VS caracteriza-se como um dos cenários desse aprendizado transformador ${ }^{10}$.

Tendo em vista esta proposta educacional do PET-Saúde/VS, que tenta impulsionar o contato interprofissional e integrar os atores envolvidos com a realidade dos serviços de saúde, idealizouse a realização de uma avaliação desse programa, envolvendo seus atores. Compreende-se que a aproximação ensino-serviço é imprescindível para que se materializem transformações efetivas na formação de recursos humanos no campo da saúde no Brasil, bem como para que a produção de conhecimento cause impacto significativo nos indicadores de saúde e na qualidade da atenção prestada no Sistema Único de Saúde (SUS) ${ }^{10}$. Nesse sentido, uma avaliação pode ajudar a determinar as mais diversas características de um programa, seus pontos fortes e fragilidades.

Historicamente, a avaliação de programas tem sua origem em Ralph Tyler, a partir de estudo realizado em 1930 sobre a correlação entre currículos do nível secundário e desempenho subsequente dos alunos na faculdade. Este estudo durou oito anos, por meio do acompanhamento de uma leva de alunos americanos nos quatro anos do nível Médio e nos quatro da faculdade. Tyler pode caracterizar os pontos fortes e fracos das propostas curriculares, correlacionando-os aos objetivos declarados. Dessa forma, extrapolou as fronteiras da avaliação, até aquele momento circunscrita à simples medição, e inaugurou o que, posteriormente, seria chamado de avaliação formativa ${ }^{5}$. 
Atualmente, a avaliação de programas e serviços insere-se, assim como o planejamento em saúde, na temática da gestão em saúde coletiva. A avaliação acontece quando os pressupostos e as ações de um determinado programa ou serviço são confrontados com a prática, gerando informações que trazem esclarecimentos e subsidiam decisões. Nesse sentido, gestão, avaliação e planejamento podem ser considerados instâncias muito próximas e convergentes ${ }^{5}$.

\section{Metodologia}

Na Universidade Federal de Santa Maria, o PET-Saúde/VS é desenvolvido por: cinco tutores docentes da UFSM (Medicina, Farmácia, Odontologia, Terapia Ocupacional e Veterinária), seis preceptores (profissionais de serviços municipais de saúde e da $4^{\mathrm{a}} \mathrm{CRS}$ ) e 24 alunos de oito cursos de graduação (Enfermagem, Farmácia, Fisioterapia, Medicina, Odontologia, Terapia Ocupacional, Serviço Social e Veterinária). A avaliação foi voltada para os estudantes e preceptores (total de trinta pessoas) da equipe. A participação dos profissionais dos serviços de saúde públicos no processo ensinoaprendizagem dos alunos, mediada pelo tutor, é relevante no desenvolvimento de conhecimentos e habilidades, pois contribui com a formação de competências necessárias às práticas de saúde desta área².

Este trabalho apresenta, de forma qualitativa, o relato de uma experiência do processo de organização do PET-Saúde/VS. Sendo assim, utilizou-se uma ficha estruturada para avaliação do PET-Saúde/VS da UFSM, a qual fazia parte da metodologia do programa como estratégia para reorganização e planejamento das atividades, e foi solicitada, aos atores participantes do PET-Saúde/ VS da UFSM, na reunião de toda a equipe do mês de março/2014.

A ficha foi preenchida pelos próprios atores participantes do PET-Saúde/VS da UFSM sem entrevista. Os acadêmicos bolsistas e preceptores participantes do PET-Saúde entregaram as respostas de forma anônima. A avaliação consistia em perguntas abertas dirigidas a preceptores e estudantes, envolvendo a contribuição do PET-Saúde/VS para formação, facilidades e dificuldades encontradas. Também foi abordada a contribuição do PET-Saúde/VS para o serviço (se o participante fosse preceptor) ou a contribuição do PET-Saúde/VS para o cotidiano acadêmico (se o participante fosse estudante).

Os dados foram digitados e avaliados utilizando-se a técnica do Discurso do Sujeito Coletivo $(D S C)^{11}$, que consiste em um conjunto de procedimentos de tabulação e organização de dados discursivos. Este método se destina a tornar mais claras e expressivas as representações sociais. Com o sujeito coletivo, os discursos não se anulam ou se reduzem a uma categoria comum unificadora, já que o que se busca fazer é, precisamente, o inverso, ou seja, reconstruir, com pedaços de discursos individuais, como em um quebra-cabeça, tantos discursos-síntese quantos se julgue necessário para expressar uma dada "figura", um dado pensar ou uma representação social sobre um fenômeno"11.

\section{Resultados e discussões}

Foram analisadas todas as respostas dos 28 atores (quatro preceptores e 24 estudantes) participantes deste processo, das quais se obtiveram ideias centrais, representativas do discurso coletivo para cada uma das questões.

Em relação à contribuição do PET-Saúde/VS para formação, destacaram-se três ideias centrais: houve diferentes contribuições do PET-Saúde/VS no que se refere ao ensino, à pesquisa e à extensão, demonstrando que o programa contribuiu significativamente para a formação integral em saúde coletiva. Essas ideias podem ser visualizadas no Quadro 1. 
Quadro 1. Ideia central e discurso do sujeito coletivo dos atores em relação à questão "Contribuição do PET Vigilância na sua formação". UFSM, 2014.

\begin{tabular}{|c|c|}
\hline Ideia central & Discurso do Sujeito Coletivo \\
\hline (1) Ensino & $\begin{array}{l}\text { proporcionou aprendizado em VS; contribuiu significativamente para a formação visando a saúde coletiva } \\
\text { e o SUS; informações sobre o sistema de saúde; o PET vai engrandecer meu currículo, como também me } \\
\text { proporcionar conhecimentos que sem ele não teria a oportunidade de aprender; contato com a academia; } \\
\text { o PET tem contribuído no sentido de ampliar o meu conhecimento da área da saúde, por meio da parceria } \\
\text { ensino/serviço onde me é oportunizado esse contato com os serviços de saúde do município; troca de } \\
\text { informações e conhecimento com alunos e professores de áreas diferentes da minha; aperfeiçoamento e } \\
\text { construção teórica sobre questões de vigilância em saúde; construção de conhecimento aliando a teoria e a } \\
\text { prática entre a comunidade acadêmica e o trabalho no SUS; redigir textos e trabalhos com maior facilidade; } \\
\text { entender o contexto do meu curso dentro da vigilância em saúde; argumentar sobre a saúde pública com maior } \\
\text { conhecimento; desenvolvimento de atividades extracurriculares; conhecer melhor a vigilância epidemiológica, } \\
\text { saber como é o funcionamento, sua importância para saúde; conhecimento mais profundo sobre os outros } \\
\text { cursos; o trabalhar em equipe com formações diversas tem sido de grande crescimento acadêmico e penso } \\
\text { que fará diferença quando estiver no campo de trabalho como profissional; ter oportunidade de trabalhar com } \\
\text { estudantes de outros cursos de forma multiprofissional e assim aprender sobre as outras profissões; }\end{array}$ \\
\hline (2) Pesquisa & $\begin{array}{l}\text { possibilidade de desenvolver e acompanhar trabalhos científicos; ensina na construção de projetos; contribui } \\
\text { com a publicação de "projetos"; construção de trabalhos científicos; nos permite vivenciar a elaboração do } \\
\text { processo de pesquisa juntamente com os colegas e professores dos outros cursos de graduação. }\end{array}$ \\
\hline (3) Extensão & $\begin{array}{l}\text { mostra a realidade da Atenção Básica em Saúde (ABS) e VS; vivências do trabalho interdisciplinar; } \\
\text { proximidade com os serviços; aprender a ter desenvoltura para lidar com desafios que enfrentará no futuro } \\
\text { profissional; proporcionou experiência em VS; propiciar o trabalho com uma equipe multidisciplinar; permitir } \\
\text { conhecimento (antecipado) dos serviços de saúde e do seu funcionamento, melhorando assim minha } \\
\text { atuação profissional; aprender a conviver com diferentes áreas da saúde e com as dificuldades encontradas } \\
\text { pelos profissionais que atuam na saúde do município; importante principalmente em relação ao trabalho } \\
\text { multiprofissional dificilmente encontrado durante a graduação, pois nos permite vivenciar a elaboração do } \\
\text { processo de trabalho juntamente dos colegas e professores dos outros cursos de graduação; está contribuindo } \\
\text { positivamente, pois a experiência do contato com estudantes de diferentes cursos, com profissionais de } \\
\text { outras áreas está nos auxiliando a crescer tanto profissionalmente como pessoalmente; contribuição para o } \\
\text { melhor entendimento dos serviços de VS e gestão dos mesmos nas três esferas (federal, estadual e municipal); } \\
\text { vivência e compartilhamento com os alunos da graduação de campo e núcleo profissional; participação } \\
\text { muito significativa, me fez ver uma nova possibilidade de trabalho dentro da minha profissão, sendo decisivo } \\
\text { no que se refere ao meu futuro profissional e ambiçães futuras; oportunidade de participação em eventos } \\
\text { deliberativos; possibilitou conhecer a rede e o modo como funciona e se estrutura o sistema de saúde, seus } \\
\text { pontos fortes e fracos; proporcionou a oportunidade de interagir e trabalhar com alunos, professores, tutores } \\
\text { e preceptores de outros cursos e que atuam diretamente no serviço de saúde ou na vigilância epidemiológica; } \\
\text { temos uma visão de campo a partir dessa interação, passamos a entender o processo de formação de trabalho } \\
\text { e os desafios que cada profissional enfrenta na sua área, não nos restringindo apenas ao nosso curso; o PET } \\
\text { trouxe um leque de possibilidades de futura atuação profissional, além de possibilitar experiências que do } \\
\text { contrário, provavelmente não teria acesso; construção de conhecimento aliando a teoria e a prática entre o } \\
\text { trabalho no SUS e a comunidade acadêmica. }\end{array}$ \\
\hline
\end{tabular}

Observa-se que as diferentes experiências de ensino-aprendizagem oportunizadas pelo PETSaúde/VS possibilitam a formação de um profissional de saúde crítico, reflexivo, proativo, preparado para atuar em equipe e no mercado de trabalho, conforme Souza e colaboradores ${ }^{12}$. A pesquisa, por sua vez, é fundamental no processo de extensão dos produtos do conhecimento à sociedade, pois a prestação de qualquer tipo de serviço à comunidade social, que não decorre do conhecimento da objetividade dessa comunidade, é mero assistencialismo ${ }^{13}$. Conforme Rodrigues et al. ${ }^{14}$, a interação ensino-serviço-comunidade é fundamental para a formação de profissionais comprometidos com a proposta do SUS, proporcionando-Ihes um contato direto com os problemas da população e instrumentalizando-os para intervirem, de forma eficaz, com ações coletivas por meio de uma educação preventiva em saúde pública.

Para o serviço, as contribuições descritas pelos preceptores foram divididas em duas ideias centrais: troca de experiências multiprofissionais e conhecimento/pesquisas, conforme pode ser visualizado no Quadro 2. 
Quadro 2. Ideia central e discurso do sujeito coletivo dos preceptores em relação à questão "Contribuição do PET Vigilância para o serviço". UFSM, 2014.

\begin{tabular}{|c|l|}
\hline $\begin{array}{c}\text { (1) troca de } \\
\text { experiências } \\
\text { multiprofissionais }\end{array}$ & $\begin{array}{l}\text { integração dos alunos no serviço, compondo as equipes de trabalho; presença dos alunos no serviço, } \\
\text { contribuindo com suas experiências; importância da integralidade na atenção à saúde; integração } \\
\text { com os grupos; minha presença nos grupos realizados na unidade se tornou mais frequente; no } \\
\text { cotidiano do trabalho, o PET tem proporcionado que possamos ter um olhar de "fora" sobre o nosso } \\
\text { trabalho, amplia a perspectiva de trabalho na direção do planejamento das ações; momentos de } \\
\text { reflexão sobre o trabalho nos encontros entre alunos, preceptores e tutores. }\end{array}$ \\
\hline \multirow{2}{*}{$\begin{array}{c}\text { (2) conhecimento/ } \\
\text { pesquisas }\end{array}$} & $\begin{array}{l}\text { presença dos alunos no serviço, contribuindo com seus conhecimentos; ampliar meus conhecimentos } \\
\text { sobre ABS; relembrar conceitos de epidemiologia; troca de informações com estudantes de diversas } \\
\text { áreas que sempre tem a acrescentar uma opinião ou um método de trabalho diferente; discussão } \\
\text { de temas atuais; fazer pesquisas a partir da realidade do cotidiano no SUS, ou seja, construção de } \\
\text { conhecimento a partir da prática. }\end{array}$ \\
\hline
\end{tabular}

A partir dos relatos descritos no Quadro 2, verifica-se que, no decorrer das ações previstas para os grupos tutoriais, a convivência dos preceptores com os docentes e discentes de graduações distintas desafiou a todos para o desenvolvimento de atividades que não eram realizadas em suas rotinas. Assim, todos aprendem sobre, de e com o outro, a fim de permitirem a melhoria dos resultados em saúde ${ }^{15}$. Ou seja, a Educação Interprofissional em Saúde (EIP) possibilita que os estudantes, docentes e preceptores aprendam juntos e ampliem o seu conhecimento e as suas experiências ${ }^{15}$, uma vez que possibilitam oportunidades para o desenvolvimento de aprendizagens compartilhadas ${ }^{16}$.

Já para o cotidiano acadêmico, a percepção dos estudantes pode ser observada pelo discurso: "Passamos a ampliar o olhar a partir das vivências, deixamos de ser meros expectadores e passamos a atuar como protagonistas, interagindo com outras profissões, associando teoria à prática!"

Tanto no serviço quanto no cotidiano acadêmico, refletir sobre as práticas de saúde implica refletir sobre a formação e o desenvolvimento dos profissionais da área, mediante os modos de ensinar e aprender nas academias, e das formas de educar, cuidar, tratar e acompanhar as pessoas que necessitam de assistência ${ }^{17}$. A integração com outros cursos também é relatada por Leite et al. ${ }^{18}$, permitindo a troca de experiências e a aprendizagem significativa para a formação profissional.

Quanto às principais facilidades referidas pelos estudantes e preceptores no desenvolvimento das atividades realizadas no PET, salienta-se a afinidade com os temas abordados (disponibilidade de um elenco diverso de assuntos a serem discutidos; compreensão das temáticas e atividades propostas; facilidade de compreender os assuntos abordados e as atividades propostas pela equipe de coordenação do PET); o uso de ferramentas de facilitação (facilidade de comunicação interpessoal, criatividade, flexibilidade de horários, disponibilidade de material no Dropbox ${ }^{\circ}$, cursos de metodologia científica) e a integração multiprofissional (integração entre alunos, preceptores, tutores; ajuda dos coordenadores e preceptores; divisão em pequenas equipes e comissões; boa convivência com os colegas; satisfação em trabalhar com pessoas). Aprender com pessoas de diferentes áreas de conhecimento parece ser uma experiência que amplia a compreensão do trabalho coletivo ${ }^{12}$.

Em contrapartida, as principais dificuldades encontradas foram aquelas relacionadas com o tempo/ organização (conciliar atividades do PET com atividades curriculares; dificuldade de organização do grupo pela falta de horários livres em meio à graduação; incompatibilidade de horários e dificuldades de horários de atendimento do serviço); nivelamento teórico (dificuldade de entender conceitos que não foram abordados no cotidiano acadêmico; dificuldade em elaborar projetos); desmotivação (problemas de comunicação referentes às reuniões e execuções de atividades); participação nas reuniões (reuniões demasiadas - reunião geral, de grupo tutorial e de comissões; reuniões gerais deveriam ser mais objetivas e resolutivas), e, por fim, falta de apoio preceptores/alunos/tutores (dificuldade de acesso a alguns preceptores; dificuldade no engajamento de todos os participantes, sobretudo os alunos; dificuldade em entrar em contato com alguns tutores). 
Percebe-se que existe uma escassez de "horários livres" nas grades curriculares dos estudantes. Além disso, existem dificuldades de disponibilidade de horários comuns entre estudantes e professores oriundos de vários cursos de graduação. Também existem limitações para a inserção de estudantes no serviço e de preceptores na universidade em função da coincidência dos horários das atividades acadêmicas e do funcionamento dos serviços. É consenso de que, para se estabelecer um trabalho coeso e eficiente, são necessários: o envolvimento de todos, a busca pela satisfação no trabalho, o treinamento e o desenvolvimento de competências, a criação de sinergia entre os participantes, a definição de papéis, além do acompanhamento e da avaliação de resultados ${ }^{16}$.

\section{Conclusões}

A avaliação do programa em etapa intermediária do seu desenvolvimento possibilitou a valorização de estudantes e preceptores como construtores deste processo de ensino-aprendizagem. O instrumento de avaliação permitiu analisar o impacto da participação do PET-Saúde/VS da UFSM no desenvolvimento das habilidades e competências dos diferentes atores do Programa e o processo de ensino, pesquisa e extensão durante o período.

Com estes dados, obteve-se uma ferramenta orientada para a ação e a aprendizagem. Além disso, com o conhecimento das opiniões dos atores, em relação ao PET-Saúde/VS, das suas facilidades e dificuldades, encontraram-se subsídios para se realizarem as mudanças no planejamento para o segundo ano do programa. Assim, poderemos tanto melhorar as atividades em andamento quanto planejar o futuro e orientar a tomada de decisões.

Enfim, observou-se que, ao mesmo tempo em que se reconhece a importância da formação multiprofissional para ABS na sua integralidade, esbarra-se na dificuldade de se reunirem estudantes e professores de diversos cursos devido à incompatibilidade de horários. Da mesma forma que a almejada interação da academia com o serviço depara-se com a incompatibilidade no horário de funcionamento deste com os horários dos cursos de graduação da área da saúde.

\section{Colaboradores}

Liziane Maahs Flores responsabilizou-se pela fase de tabulação, análise dos dados, redação e revisão do artigo; Ana Luiza Trindade responsabilizou-se pela fase de tabulação e análise dos dados; Daiane Rodrigues de Loreto responsabilizou-se pela fase de análise dos dados, redação e revisão do artigo; Beatriz Unfer responsabilizou-se pela fase de elaboração do instrumento de avaliação, coleta de dados e revisão do artigo; Marinel Mór Dall'Agnol responsabilizou-se pela fase de análise dos dados e revisão do artigo.

\section{Referências}

1. Ministério da Saúde (BR). Portaria Interministerial $n^{\circ} 1.802$, de 26 de agosto de 2008. Institui o Programa de Educação pelo Trabalho para a Saúde - PET-Saúde. Diário Oficial União. 25 jun 2007.

2. Ministério da Saúde (BR). Portaria Interministerial n 422, de 3 de março de 2010. Estabelece orientações e diretrizes técnico-administrativas para a execução do Programa de Educação pelo Trabalho para a Saúde (PET-Saúde), instituído no âmbito do Ministério da Saúde e do Ministério da Educação. Diário Oficial União. 5 mar 2010;Seção 1:53. 
3. Ministério da Saúde (BR). Portaria $n^{\circ} 1.378$, de 9 de julho de 2013. Regulamenta as responsabilidades e define diretrizes para execução e financiamento das ações de Vigilância em Saúde pela União, Estados, Distrito Federal e Municípios, relativos ao Sistema Nacional de Vigilância em Saúde e Sistema Nacional de Vigilância Sanitária. Diário Oficial União, 10 jul .2013; Seção 1:48.

4. Conselho Nacional de Secretários de Saúde - CONASS. Vigilância em saúde: parte 1. Brasília, DF: Conselho Nacional de Secretários de Saúde; 2011. (Para entender a gestão do SUS/2011, vol. 5).

5. Furtado JP. Avaliação de programas e serviços em saúde. In: Campos GWS, Bonfim JRA, Minayo MCS, Akerman M, Drumond Júnior M, Carvalho YM, organizadores. Tratado de saúde coletiva. 2a ed. São Paulo: Hucitec; 2012. p. 765-93.

6. Biblioteca Virtual em Saúde. DeCS - Descritores em Ciências da Saúde. São Paulo; Bireme; 2014 [acesso em 28 ago 2014]. Disponível em: http://www.decs.bvs.br

7. Instituto Brasileiro de Geografia e Estatística - IBGE. Censo demográfico 2010. Rio de Janeiro: Instituto Brasileiro de Geografia e Estatística; 2010 [acesso 28 ago 2014]. Disponível em: http://www.ibge.gov.br/home/estatistica/populacao/censo2010/

8. Albuquerque IM, Pasqualotto AS, Trevisan ME, Gonçalves MP, Badaró AFV, Moraes JP et al. Role of physiotherapy in the rehabilitation of survivors of the kiss nigthclub tragedy in Santa Maria, Brazil. Physiotherapy. 2013;99(4):269-70. http://dx.doi.org/10.1016/j. physio.2013.07.001

9. Albuquerque VS, Gomes AP, Rezende CHA, Sampaio MX, Dias OV, Lugarinho RM. A integração ensino-serviço no contexto dos processos de mudança na formação superior dos profissionais da saúde. Rev Bras Educ Med. 2008;32(3):356-62. http://dx.doi. org/10.1590/S0100-55022008000300010

10. Linhares MSC, Freitas CASL, Teixeira AKM, Dias RV, Flor SMC, Soares JSA et al. Programa de educação para o trabalho e vigilância em saúde. Trab Educ Saúde. 2013;11(3):679-92. http://dx.doi.org/10.1590/S1981-77462013000300012

11. Lefèvre F. Discurso do sujeito coletivo: um novo enfoque em pesquisa qualitativa (desdobramentos). Caxias do Sul: EDUCS; 2003.

12. Souza PL, Pereira CS, Nogueira MLS, Pereira DB, Cunha GM, Möler FO. Projetos PET-Saúde e Educando para a Saúde: construindo saberes e práticas. Rev Bras Educ Med. 2012;36(1 Supl 1):172-7. http://dx.doi.org/10.1590/S0100-55022012000200024

13. Severino AJ. Educação e universidade: conhecimento e construção da cidadania. Interface (Botucatu). 2002;6(10):117-24. http://dx.doi.org/10.1590/S141432832002000100015

14. Rodrigues AAAO, Juliano IA, Melo MLC, Beck CLC, Prestes FC. Processo de interação ensino, serviço e comunidade: a Experiência de um PET-Saúde. Rev Bras Educ Med. 2012;36(1 Supl 2):184-92. http://dx.doi.org/10.1590/S0100-55022012000300027

15. World Health Organization. Framework for action on interprofessional education \& collaborative practice. Geneva: WHO; 2010.

16. Aguilar-da-Silva RH, Scapin LT, Batista NA. Avaliação da formação interprofissional no Ensino Superior em Saúde: aspectos da colaboração e do trabalho em equipe. Avaliação (Campinas). 2011;16(1):167-84. http://dx.doi.org/10.1590/S1414-40772011000100009

17. Araujo FC, Falcon EBS, Rodrigues GM, Freitas LC, Dutra CDT, Pires CAA. O aprender e o orientar na atenção primária: relato de experiência de um semestre de atividades no PET-Saúde. Rev Bras Educ Med. 2012;36(1 Supl 2):164-8. http://dx.doi.org/10.1590/ S0100-55022012000300024

18. Leite MTS, Rodrigues CAQ, Mendes DC, Veloso NS, Andrade JMO, Rios LR. O Programa de Educação pelo Trabalho para a Saúde na formação profissional. Rev Bras Educ Med. 2012;36(1 Supl 1):111-8. http://dx.doi.org/10.1590/S0100-55022012000200015 
O PET-Saúde/VS da Universidade Federal de Santa Maria, Rio Grande do Sul, Brasil, surgiu para realizar ações de vigilância emergenciais às vítimas do desastre em casa noturna de Santa Maria e a análise situacional em saúde do município. Entendendo que a avaliação é essencial para o planejamento, a implementação e o funcionamento de qualquer programa, elaborou-se um questionário para avaliar o PET-Saúde/VS por sua equipe. As perguntas tratavam da contribuição do programa para a formação acadêmica e/ou serviço, bem como facilidades e dificuldades encontradas pela equipe. Os dados foram avaliados de forma qualitativa, utilizando-se o método do Discurso do Sujeito Coletivo. O programa contribuiu para o aperfeiçoamento do ensino, da pesquisa e da extensão, possibilitando vivências, desenvolvimento acadêmico e contatos multiprofissionais. A integração multiprofissional e a comunicação interpessoal foram as facilidades destacadas, enquanto o desnivelamento teórico e a conciliação de horários foram as principais dificuldades.

Palavras-chave: Vigilância em saúde pública. Educação em saúde. Serviços de integração docente-assistencial.

\section{Evaluation of the Education by Work for Health Program PET-Health/Surveillance Public Health by their actors}

The PET-Health/SH of the Federal University of Santa Maria, Rio Grande do Sul State, Brazil, was created to execute emergency surveillance to disaster victims from a Santa Maria nightclub and to conduct a situational analysis of municipal health. Because evaluation is essential for the planning, implementation, and operation of any program, a questionnaire for the assessment of PET-Health/SH was drafted. The questions involved the contribution of the program to the academic development and/or service and facilities and difficulties encountered by the team. Data were qualitatively analyzed using the Collective Subject Discourse Method. The program contributed to an improvement in teaching, research, and extension and enabled life experiences, academic development, and multi-professional contacts. Multi-professional integration and interpersonal communication facilities were highlighted, while theoretical deflection and schedules were the main difficulties.

Keywords: Public health surveillance. Health education. Teaching care integration services.

\section{Evaluación del Programa de Educación para el Trabajo para la Salud/Vigilancia en Salud Pública por sus actores}

El PET-Salud/VS de la Universidad Federal de Santa María, Rio Grande do Sul, Brasil, surgió para mantener acciones de vigilancia y de emergencia con las víctimas del desastre en el club nocturno en Santa María y para analizar la situación de salud en la municipalidad. Entendiendo que la evaluación del programa es esencial para la planificación, implementación y operación de cualquier programa, fue elaborado un cuestionario para la evaluación de la PET-Salud/VS. Las preguntas consideraron la contribución del programa para la formación académica y/o servicio, así como, las facilidades y dificultades encontradas por el equipo. Los datos se analizaron cualitativamente mediante el método del Discurso del Sujeto Colectivo. El programa contribuyó para mejorar la docencia, investigación y extensión posibilitando vivencias, desarrollo académico y contactos multiprofesionales. Las integración multiprofesional y la comunicación interpersonal fueron destacados, pero el desnivel teórico y conciliación de horarios fueron las principales dificultades.

Palabras clave: Vigilancia en salud pública. Educación en salud. Servicios de integración docente asistencial. 